\title{
Micro operators for ultra-dense network deployment with network slicing and spectrum micro licensing
}

\author{
Marja Matinmikko-Blue \\ Centre for Wireless Communications \\ University of Oulu \\ Oulu, Finland \\ marja.matinmikko@oulu.fi
}

\author{
Seppo Yrjölä \\ Nokia \\ Oulu, Finland \\ seppo.yrjola@nokia.com
}

\author{
Matti Latva-aho \\ Centre for Wireless Communications \\ University of Oulu \\ Oulu, Finland \\ matti.latva-aho@oulu.fi
}

\begin{abstract}
G emerges with ultra-dense deployments of small cell networks to address the location specific service demand of various vertical sectors. While the development of technical solutions for network densification is progressing, less attention has been paid to local operator models and location specific service offerings. Efficient local service delivery promoting innovation and competition calls for opening of the mobile market for new entrants to operate local radio access networks (RAN) and 3rd parties to integrate their services into the networks. This paper applies the recently proposed micro operator concept to local ultra-dense networks where different stakeholders can become micro operators and deploy their small cell RAN for tailored service delivery. We depict the resulting high-level architecture and enabling techniques focusing on network slicing and spectrum micro licensing. While network slicing is well addressed in $5 \mathrm{G}$ research from the mobile network operator (MNO) view for providing an end-to-end connection for 3rd parties, there is little work on how the ultra-dense small cell RAN could be operated by another stakeholder. Micro operators could offer the RAN or end-to-end network slice that comprises of network elements belonging to different stakeholders with the help of local spectrum micro licensing with quality guarantees.
\end{abstract}

Keywords-5G; architecture; mobile network operator; infrastructure; spectrum sharing;

\section{INTRODUCTION}

Future competiveness of both public services and enterprises relies on cost-effective access to high capacity networks to create new applications and business models for production, distribution and selling of products and services [1]. This will require gigabit level wireless connectivity to serve various public places, such as schools, stations, authority buildings, universities, hospitals, malls, and stadiums as well as closed premises including factories, power plants, and enterprises [1]. Cost-efficient service delivery calls for changes in the traditional mobile market regulations and stakeholder roles by allowing multiple tenants to operate over the same shared infrastructure and by integrating 3rd party provided tailored services into the network [2].

While the majority of indoor mobile traffic today is carried via wireless local area (WLAN) networks, the role of ultradense networks in the cellular 5G domain has gained attention to meet the growing mobile data demand [3]-[4]. Network

Authors would like to acknowledge the funding from Business Finland in uO5G project. infrastructure densification with the introduction of a large number of small cells is under extensive study as a solution to increase capacity and service quality in local high demand areas. 5G architectural developments including virtualized network functions and operations in higher frequency bands are specifically addressing this development unlike the previous network generations that aimed at providing mobile connectivity for wide areas [5].

Successful deployment of ultra-dense small cell networks is not only a technical matter but involves market and regulatory decisions to determine who can operate the new small cell RAN and under which rules and conditions, see the discussion in [4]. Existing spectrum authorization models for ultra-dense networks include overlay deployment of small cells on MNO's exclusively licensed bands with its other cell layers and alternatively in unlicensed bands with different rules for operations. This traditional logic adds the ultra-dense networks as a new resource layer into MNOs' heterogeneous networks. While sharing-based operational models are discussed in regulation [6], the existing spectrum authorization models have prevented non-MNOs from deploying cellular RANs due to the lack of locally licensed spectrum. Thus, they can currently only use unlicensed bands resulting in lack of quality guarantees. A new licensing regime for ultra-dense networks was proposed in [4] where the facility owner would gain access to all spectrum provided that it opens its indoor network to MNOs.

The role of network slicing has become important in $5 \mathrm{G}$ to expand the market by allowing 3rd parties to serve vertical sectors' needs [7]. A network slice broker role has emerged to manage the transactions and technical operations of network slicing [8]. The RAN aspects of network slicing are discussed in [7] focusing on how to provide traffic isolation and radioelectrical isolation between different network slices. However, the underlying assumption in network slicing so far has been that the RAN is operated by the MNO and the local $3^{\text {rd }}$ party service provider buys the network slice from the MNO. This paper presents the deployment of ultra-dense small cell RAN in specific locations using the recently proposed micro operator concept [9]-[11]. It allows new stakeholders to take the micro operator role and deploy an ultra-dense small cell RAN in specific locations and establish a fully functional network in collaboration with various infrastructure service providers (e.g. MNOs and network vendors) for tailored service delivery. 
The rest of this paper is organized as follows. First, the micro operator concept is described considering its architecture and enabling technologies. The role of network slicing is then discussed as a key enabler for integrating local micro operators into the big mobile connectivity market followed by spectrum considerations that highlight the need for new local spectrum authorization models. Finally, future challenges are presented.

\section{MICRO OPERATORS FOR ULTRA-DENSE NETWORK DEPLOYMENT}

The concept of micro operators has emerged to open the mobile market for locally deployed wireless networks in specific premises [9]-[11]. The micro operator concept allows different stakeholders to establish local small cell networks and offer context related services and content with quality guarantees [9]. A micro operator can serve existing MNOs by offering them local capacity in high demand areas, its own restricted customer set, or a mix of both. Moreover, $3^{\text {rd }}$ parties such as facility owners and users could have an important role in offering context related services and content in local areas in different verticals through local small cell communication infrastructure provided by the micro operator.

Deployment of local ultra-dense small cell networks by micro operators requires changes to the traditional operator models and regulations to allow new agile players to deliver new services [10], [12]. Micro operator use cases include deployments of ultra-dense small cell RANs in different locations such as factories, campuses, malls, and sports arenas [9]. An overview of use cases, benefits, enabling technologies and future challenges for micro operator is presented in Fig. 1. With the help of network slicing and spectrum sharing techniques, the micro operator can rapidly respond to local needs and provide high connectivity services. A network slice can be tailored to support specific applications/services delivered over micro operator's network where the RAN part of the slice is from the micro operator and other parts can be from the owner of other physical network infrastructure.

In fact, a fundamental rethinking of the mobile network operational principles is needed to address the micro operator requirements for diverse locations, services, use cases, and business models. The ability to identify and capture network resources and capabilities in the given geographical area can be combined with the ability to enable usage at the needed service level for the use case. The micro operator ecosystem enables any party that would need operator level wireless capacity in selected locations to build their own network solution or take it "as-a-Service". The micro operator concept can introduce a new control point in the digital value platform through spectrum and network slice management in technology (selforganizing network (SON) and management and orchestration (MANO)), policy (marketplace) and business (as-a-Service).

Enterprises are increasingly looking for $3^{\text {rd }}$ parties to provide their essential communication services, content hosting, enterprise resource management (ERM), and security system and services due to outsourcing. Partners can be web service, IT system or communication service providers. These services can be offered either as cloud based Platform as a Service (PaaS) where enterprise or vertical can build their own services, or as a complete software system (SaaS). Services can be hosted within the enterprise cloud, or on a 3rd party virtualized multi-tenant cloud infrastructure. Furthermore, service providers with local presence can provide localized edge clouds to host latency sensitive, high throughput and massive/critical data applications.

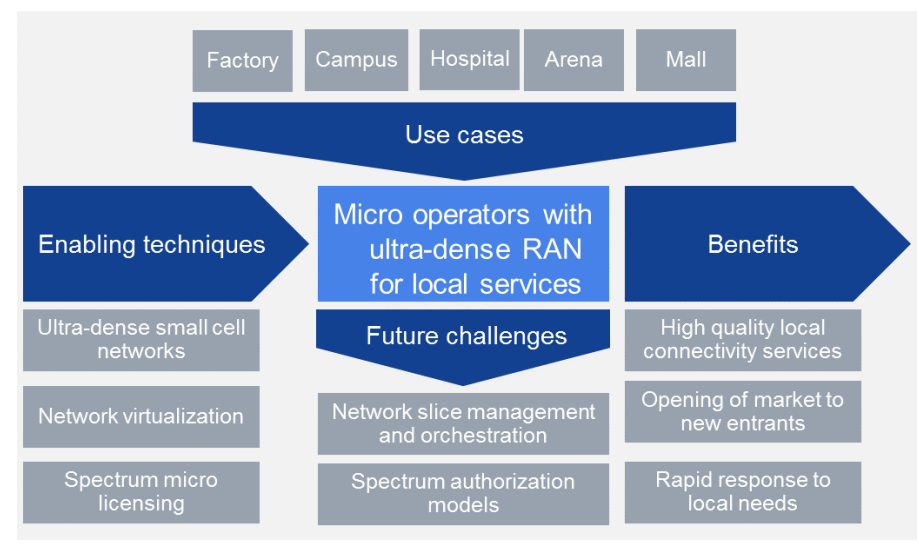

Fig. 1. Overview of use cases, benefits, enabling technologies and future challenges for micro operator

There will be several micro operator deployment scenarios as depicted in Fig. 2. In a wireless enterprise scenario, a micro operator can be a novel independent operator or IT provider offering a service bundle with in-building connectivity by running a private network. The micro operator can install and manage local wireless network and roaming contracts with MNO. Network infrastructure can be owned by the micro operator or enterprise/vertical. Alternatively in the Infrastructure as a Service (IaaS) model, a micro operator acts as a neutral host and offers multi-tenant infrastructure service with value added localized resource optimization to larger MNOs. An MNO provides applications, and manages and optimizes the service with components either instantiated in the micro operator edge cloud, or in the MNOs cloud. In the Platform-as-a-Service (PaaS) or SW-as-a-Service (SaaS) model the micro operator will operate and offer a hosted aaS functions or complete service components to MNOs. An MNO will manage end-to-end service leveraging micro operator's function and services optimized and scaled for local dynamics in demand, resources and network status. Also, a mix of a private network for restricted customer set and neutral hosting for MNOs' customers is possible.

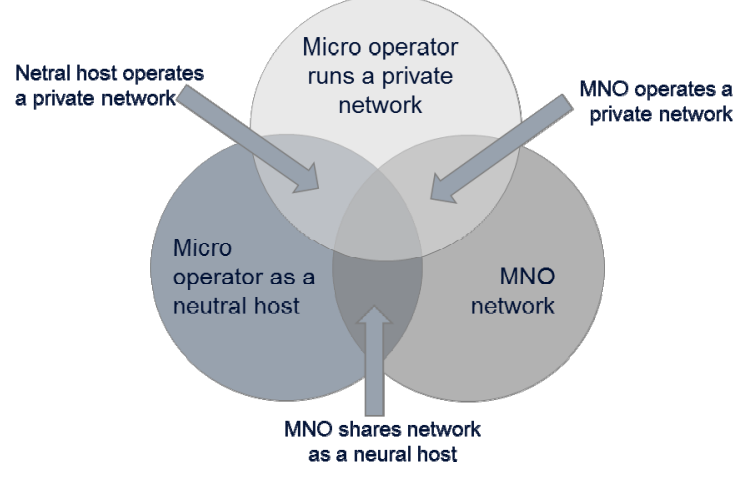

Fig. 2. Alternative micro operator deployment scenarios 


\section{ARCHITECTURE AND ENABLING TECHNIQUES}

The 5G network architecture development requires a shift from the current network of entities to a network of capabilities. Furthermore, network models will shift from the current network for connectivity to a network for services. Fig. 3 depicts a high-level network architecture where network slices are created to serve the needs of different verticals like media, industry and health in various locations such as campuses, factories and hospitals. Through service and network orchestration, it is possible to use optimized resource combinations that span over multiple stakeholders' domains for high-quality local service delivery.

There are various enabling techniques to promote local deployments of ultra-dense networks by micro operators. Fig. 4 illustrates the key enabling techniques at different network layers along with the technology evolution timeline. In the radio access layer the use of enterprise small cells to provide indoor connectivity instead of macro cells is increasing, and the virtualization of RAN allows more efficient resource usage for local service delivery and can ultimately result in stand-alone $5 \mathrm{G}$ radios operating independently of the MNO network. Processing and storage are moving from centralized solutions to local edge clouds where the current caching and local computing will evolve through cloud RAN and dynamic RAN sharing towards full support of latency critical local applications at the cell edge. Core network evolves from cloud network where one network serves all services and devices, into horizontal slicing across network domains, i.e. from radio access through edge to core and vertical slicing where network is offered as a service for $5 \mathrm{G}$ verticals with different device segments and customer segments. Ultimately, horizontal and vertical slicing will allow service and consumer specific customization in parallel with mobile broadband offerings.

Network management and operations include cognitive features to dynamically reconfigure networks according to local spectrum availability. Network management will evolve to dynamic orchestration of network slices across multiple stakeholders' domains. Digital value platforms evolve from platforms hosted on the local edge cloud to brokerage of various network resources. In the security and privacy layer, security functions are applied at the network edge to protect the network by reacting to threats locally. Digital trust is needed to create brokerage across multiple stakeholders' domins, which could be accomplished with e.g. blockchain. Security autonomics will allow the networks to monitor and adjust the slices and virtualized elements according to detected security threats without human intervention.

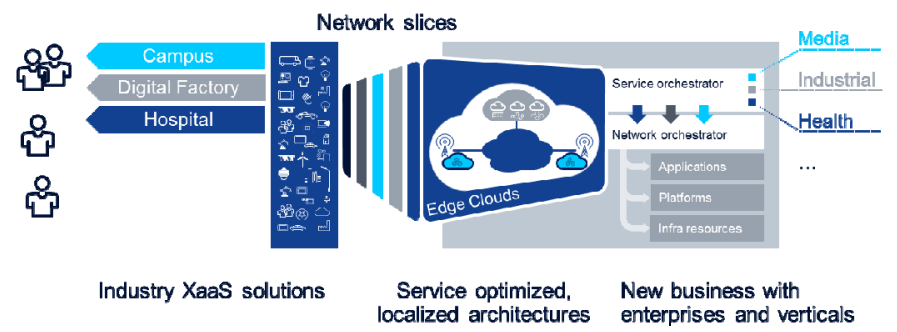

Fig. 3. 5G architecture addressing different industry and multi-tenancy use cases

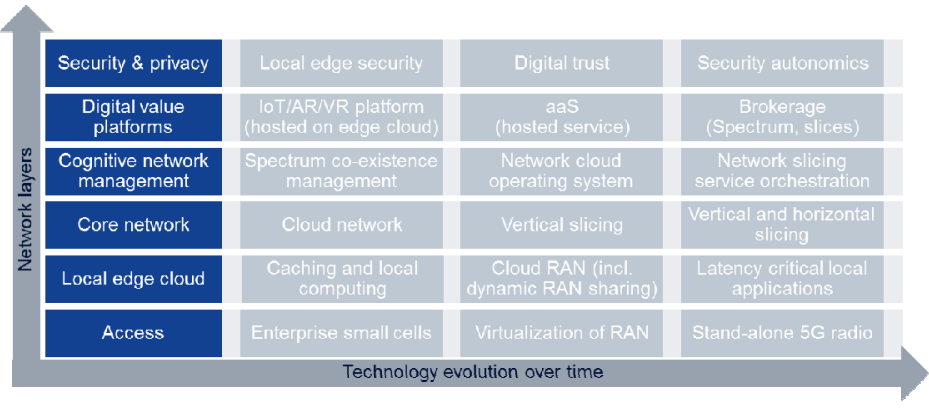

Fig. 4. Evolution of enabling techniques for micro operators

\section{ROLE OF NETWORK SLICING}

Network slicing offers a way to meet the diverse use case requirements and exploit the benefits of a common network infrastructure [13]-[15]. It enables operators to establish different capabilities, deployments, and architectural flavors for each use case or service group and run multiple network instances in parallel as depicted in Fig. 3. Each slice has its own isolated set of resources and can be based on different architectural principles that provide appropriate means to keep networks separate, and if needed the slices can share resources. A network slice can span across multiple stakeholders' domains where the micro operator could deploy an ultra-dense RAN in a specific location. Slicing allows operators to provide services by abstracting the slice functionality and expose capabilities to $3^{\text {rd }}$ party service providers through open application programming interfaces (APIs). User equipment can be served by any control plane (CP)/user plane (UP) slice and multiple slices at a time, depending on, e.g., the user equipment (UE) capabilities and application. Definition of selection principles, a multi-dimensional descriptor (e.g., application, service) configured in the UE and reported to the network assists the network to select a particular slice [16].

Software-defined networking (SDN) and network function virtualization (NFV) will play an important role in the shift to network slicing, see e.g. [13]. SDN concept logically decouples the network control function from the network forwarding function and enables policy driven dynamic programmability of abstracted underlying infrastructure, e.g., in defining and controlling the connectivity, quality of service (QoS) and associated security rules. SDN works hand-in-hand with virtualization that will enable separation of the software from the hardware and offer the possibility to instantiate many functions on a common infrastructure leveraging commodityof-the-shelf (COTS) hardware. Furthermore, software defined virtual private networking (SD-VPN) enables virtual enterprise model that does not have any formal owned location or facilities through using SDN policy controls to create secure, SW based tunnels overlaid on any IP network to provide connections between location. Micro operators could utilize this across multiple enterprise clients to virtually separate client operations from one another. SD-VPN can coordinate among multiple MNOs and IT providers to manage inside-enterprise deployment, possible roaming agreements and WLAN access. Softwarization of the network with advances in analytics and machine learning will enable high level of automatization in development, operations and optimization. With this approach, 
the infrastructure can be efficiently shared by different tenants and provide different services. Resulting network elasticity and scalability enable network and resource usage adaptation to required capacity and service level on demand which improves business agility while reducing CAPEX and OPEX. Furthermore, software-based network can open the ecosystem to new players, and accelerate time to market by reducing service creation and activation times.

In the architecture scenario, the service orchestrator acts as the logical interface between network and business applications. It provides an abstraction of the network towards applications and interfaces for agile service creation and optimization, and expose actionable network insights to application and content providers, enterprises, and industry verticals. The 3GPP service capability exposure function (SCEF) within the trust domain provides means for an operator to expose selected service capabilities via APIs and facilitating for e.g., authentication and authorization, policy enforcement, discovery, assurance, accounting and abstraction [14]. In a Network Cloud OS concept, network services are composed using a library of functions hosted in the cloud and chained together to create an end-to-end service. Network OS provides centralized network abstraction that decouples underlying network element domain from network applications, interfaces with network consumers and allows the network to be programmed. NW OS supports multi-tenancy requirement by the creation of network slices. In network slicing architecture, the MANO layer supports design, creation, and activation of individual slices on the common infrastructure. It is enhanced using analytics and machine learning. In addition, analytics are exploited to optimize the infrastructure resources within a slice, and across different slices sharing the same infrastructure.

The infrastructure layer hosts physical and virtual resources needed to create end-to-end network slices. These include both virtualization software and hardware comprised of memory, compute, storage, and networking resources. To leverage network slicing properly, the individual segments (radio access, transport, metro, core, edge cloud, central cloud), which were formerly treated separately, must be managed, operated and optimized as a whole across the entire network. The embedding of the cloud in the network edge provides the optimum performance (throughput and latency) and economics for both the virtualized functions and any other performance critical enterprise, vertical or web service. For example, with low latency service digital factory use case will utilize user plane function at the cell edge, while traditional mobile broadband service in the campus use case will use UP function at core cloud. The emergence of micro operators with new global-local cloud paradigm with localized edge resources will allow security functions to be applied at the network edge, where they can protect the network in an efficient and scalable way. Global-local distributed cloud architecture with SDN controllers will allow the network to rapidly adapt to counter the security threats. On the physical infrastructure side, enterprise small cells deployed by the micro operator, will provide access to enterprise/vertical subscribers as well as to visitors from outside MNO network. These multi-technology, multi-band, multi-tenancy capable radios can simplify enterprise WLAN deployment with reduced CAPEX and
OPEX. Operators can offer entire virtual subnetworks to any kind of service provider or industry. This Network as a Service (NaaS) business model is an extension of existing cloud business models such as Infrastructure, Platform and Softwareas-a-Service. In the $5 \mathrm{G}$ era, the service landscape will become highly diverse with requirements to the network reaching extreme. A digital factory use case requires completely different connectivity, processing and storage resources than a network slice delivering ultra-high definition (UHD) video, or a slice that is connecting static sensors.

Moving from hierarchies to market place for the connectivity and underlying network resources (e.g., spectrum, slices) can more efficiently balance supply and demand, raise the utilization of infrastructure and ultimately maximize economic value within the industry. New roles of aggregators and brokers will emerge in moving from long term contracts to a transaction driven on-demand model. Cloud and network providers' data centers can become natural central points, representing the source and destination of much of the demand combined with context analytic-enabled optimization capabilities. Application/Service provider will be able to use sub-set of the network capabilities in a flexible, configurable and programmable manner, and to use network resources depending on their service preference. From traditional mobile operator perspective this is wholesale business as the application provider (e.g., an industry automation vendor) handles the customer relationship management (CRM) and is visible with his brand.

\section{NEED FOR SPECTRUM MICRO LICENSING}

The fundamental resource promoting or hindering the rollout of wireless networks is the availability of spectrum. When the networks are built using network slicing, the slices require radio resources for meeting the promised quality. So far, the deployment of RANs has only been possible for the MNOs due to exclusive spectrum licensing model adopted by regulators that grants a limited number of long-term spectrum access rights to deploy mobile communication networks. As the regulators in different parts of the world are committed to make new spectrum available for $5 \mathrm{G}$ [1], opening of the mobile market for ultra-dense networks deployed in specific locations by micro operators requires changes in spectrum authorization models to issue local access rights as outlined in [10] and [12].

Previously, two main spectrum authorization models considered for local ultra-dense networks were overlay deployment in MNO's exclusive bands and unlicensed bands [3]. Overlay deployment of an ultra-dense network in MNO's exclusive licensed band results in the need for intra-system interference coordination for the MNO between its macro cells and small cells using the same spectrum resources. At the same time, it limits the mobile connectivity market such that only existing MNOs can deploy and operate the ultra-dense networks. In this case, network slices would be fully owned and operated by MNOs. Under unlicensed regime, there are no QoS guarantees and possible use cases are limited to those that do not require stringent QoS. While the facility owner can control the deployment of access points within its premises and those who can access the facility, the lack of control over transmissions can result in severe service level degradation in 
unlicensed bands due to interference. It was suggested in [4] that facility owners would get access rights to all spectrum provided that they open the network to MNOs. While this approach expands the market to non-MNOs, limiting the potential license holders to one new stakeholder group (i.e, facility owners) does not promote the regulatory principles of being objective, transparent, non-discriminatory and proportionate to enhance competition and innovation.

Two recently developed spectrum sharing models approved by regulators include Licensed Shared Access (LSA) in Europe [17] and three-tier model for providing Citizens Broadband Radio Services (CBRS) in the US [18]. These models introduce mobile communications on bands shared with incumbent users. While LSA [17] provides mechanisms for protecting incumbents, it does not currently define how the additional licenses are awarded, which is a national matter. The three-tier model [18] allows MNOs and other new small scale entrants to operate CBRS on local spectrum licenses called Priority Access Licenses (PAL) in specific areas called census tracts where the license holder defines a protection area where its operation has guaranteed quality.

Efficient deployment of ultra-dense small cell RAN by micro operators calls for local spectrum micro licensing model as proposed in [10]-[12]. It defines and assigns access rights in specific locations to deploy local networks with pre-defined quality guarantees while protecting possible incumbents in the band. When deploying $5 \mathrm{G}$ networks in the millimeter-wave frequency bands, local micro licensing becomes a feasible solution as it becomes impossible to attain wide-area coverage [10]. This model allows new entrants to gain access to the spectrum in a given area to deploy the RAN with quality guarantees. The new micro licensing model can become a source of income to the regulator as spectrum is priced according to actual local demand in much shorter time-scales than the current static exclusive licenses. Fig. 5 illustrates the high-level architecture for micro licensing where the regulator defines the micro licenses, and possible incumbent spectrum users are protected from harmful interference from the micro licensees. The micro operator has a network management system with spectrum manager that controls its spectrum access according to spectrum availability information through spectrum repository and co-existence manager. The coexistence manager is a new entity to coordinate between different license holders including incumbents and micro licensees with different levels of spectrum access rights.

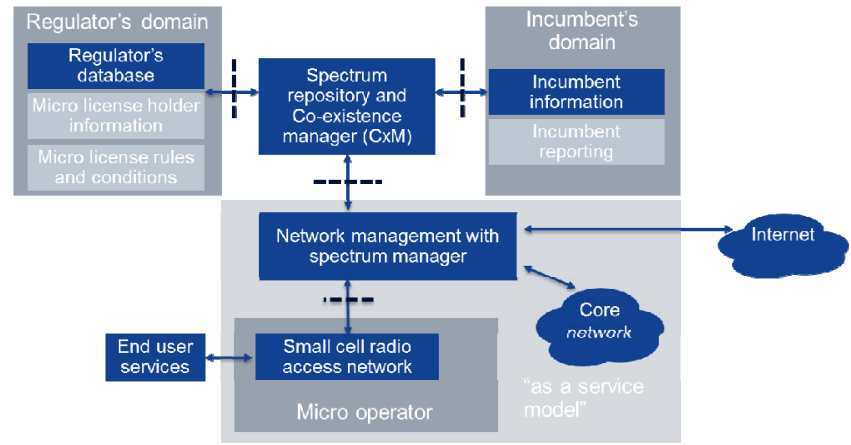

Fig. 5. High-level architecture for micro operator concept with micro licensing
The issuance of local micro licenses is specific to the frequency band in question and requires finding a balance between the dimensioning of the ultra-dense networks as well as the derivation of efficient sharing rules between local micro licensees and potential incumbent users. First, the micro operator network needs to be dimensioned such that it provides the desired coverage and capacity with quality guarantees in the specific location. This includes defining of feasible micro operator network parameters for the spectrum band in question including e.g. maximum transmission power, and the number of base stations for the network deployment. Next, the spectrum sharing conditions between the micro operator networks and possible incumbents are needed with the feasible micro operator deployment parameters. In practice, this requires defining of separation distances between the micro operators and incumbents while taking into account the aggregate interferences. Finally, sharing conditions between the different micro licensee network deployments need to be defined including separation distances between micro licensee networks in co-channel and adjacent channel situations, as initially estimated for the $3.5 \mathrm{GHz}$ band in [11], as well as any interference coordination techniques to reduce the separation distances.

In fact, a significant challenge in making the new bands available for mobile communications is the incumbent users that claim for protection from harmful interference with stringent requirements on the micro licensees limiting the $5 \mathrm{G}$ band availability. As the ultra-dense small cell deployments often take place in indoors, the building entry loss (BEL) has a significant impact on the network coverage for serving its users and also interference distances towards other networks [11]. While high BEL poses challenges on cost-efficient building of required network coverage, the indoor deployments with locally issued micro licenses can efficiently protect the incumbent users located outside the buildings, which can unlock new spectrum bands available for mobile communications on a shared basis while maintaining existing spectrum users' rights. In addition to the bands currently studied for $5 \mathrm{G}$ in the $24-86 \mathrm{GHz}$ frequency range, there are other bands that could become available for mobile communications through micro licensing. For example, the feasibility of the $10 \mathrm{GHz}$ band for $5 \mathrm{G}$ is demonstrated in [19]. The new micro licensing model is particularly efficient for allowing local access rights to micro operators as it controls the geographical coverage of the entrant mobile communication network providing protection for the incumbents. Compared to purely unlicensed model, the new micro licensing can efficiently control the aggregate interference based on the above discussed facts.

\section{FUTURE CHALLENGES}

Besides multiple benefits and enabling techniques promoting the new micro operator concept for local ultra-dense networks, there is a number of technical and regulatory challenges that need to be addressed. Key challenges related to network slicing and spectrum availability for local deployments are summarized in Fig. 6. Defining of the detailed technical architecture that integrates local ultra-dense RANs deployed by micro operators into the $\mathrm{MNO}$ driven mobile communication 
infrastructure is an open topic. It includes the identification of elements and interfaces to be opened for 3rd parties which can involve significant security challenges. Network slicing will span beyond a single MNO domain to involve multiple stakeholders including 3rd party service providers and local ultra-dense RANs deployed by micro operators, which calls for a new network slice brokerage functionality. Defining this broker in technology, business and regulatory domains is a challenge in order to enable operators to vary their charges and service level agreements within each individual slice. In fact, the decoupling of hardware resources from the network functions brings accountability challenges.

Existing regulations shaping the mobile communication market including spectrum authorization models mainly allow only MNOs to deploy cellular networks. Availability of spectrum for locally operated small cell networks by different stakeholders in specific locations is a true challenge. New $5 \mathrm{G}$ bands particularly in the millimeter wave frequencies pose new challenges on cost-efficient deployment of ultra-dense networks for high service quality while maintaining coverage without holes protecting the rights of existing incumbent spectrum users. The new micro licensing model requires careful considerations on the license awarding procedure to be objective, transparent, non-discriminatory and proportionate resulting in a potentially larger number of local license holders with requirements for protection from harmful interference. This results in the need for an efficient spectrum market place and interference coordination between micro license holders and incumbents as well as between micro license holders, which requires defining of co-existence management functions.

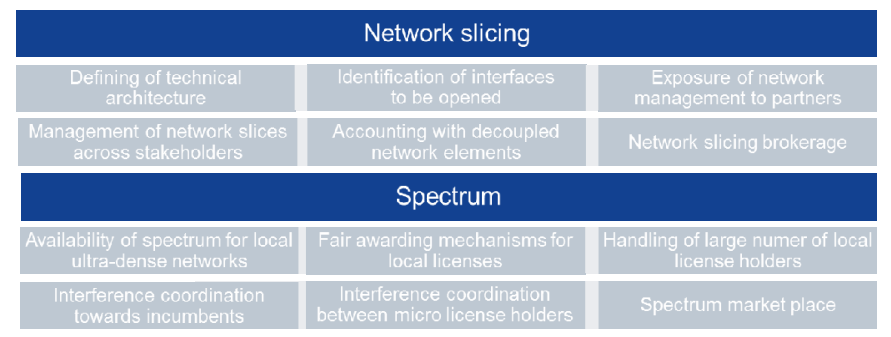

Fig. 6. Future challenges of micro operator concept

\section{CONCLUSIONS}

This article has presented the deployment of ultra-dense networks in specific locations with the newly proposed micro operator concept. It allows different stakeholders to deploy local small cell radio access networks to provide locally tailored services for verticals to complement the MNOs' mobile broadband offerings. It makes network resources locally available to service differentiation to meet the different verticals' specific needs with locally issued spectrum access rights and scale the networks according to how the demand will evolve. Through network slicing the local ultra-dense RANs operated by micro operators with locally issued spectrum access rights can be integrated into the mobile connectivity market to bring new service offerings to meet stringent and case specific customer demands.

\section{REFERENCES}

[1] European Commission. Connectivity for a competitive digital single market - Towards a European gigabit society. COM(2016) 587 final. 2016.

[2] 5GPPP. 5G innovations for new business opportunities. White paper. 5G Infrastructure Public Private Partnership. 2017

[3] R. Baldemair, T. Irnich, K. Balachandran, E. Dahlman, E. Mildh, Y. Selén, S. Parkvall, M. Meyer, and A. Osseiran, "Ultra-dense networks in millimeter-wave frequencies," IEEE Communications Magazine, pp. 202-208, 2015.

[4] J. Zander, "Beyond the ultra-dense barrier: Paradigm shifts on the road beyond 1000x wireless capacity," IEEE Wireless Communications, vol. 24, 99. 96-102, 2017.

[5] M. Agiwal, A. Roy, and N. Saxena, "Next generation 5G wireless networks: A comprehensive survey," IEEE Communications Surveys \& Tutorials, vol. 18, 1617-1655, 2016.

[6] M. Mustonen, M. Matinmikko, O. Holland, and D. Roberson, "Process model for recent spectrum sharing concepts in policy making," Telecommunications Policy, vol. 41, pp. 391-404, June 2017.

[7] O. Sallent, J. Perez-Romero, R. Ferrus, and R. Agusti, On radio access network slicing from a radio resource management perspective," IEEE Wireless Communications, vol. 24, pp. 166-174, 2017.

[8] K. Samdanis, X. Costa-Perez, and V. Sciancalepore, "From network sharing to multi-tenancy: The 5G network slice broker," IEEE Communications Magazine, vol. 54, pp. 32-39, 2016.

[9] M. Matinmikko, M. Latva-aho, P. Ahokangas, S. Yrjölä, and T. Koivumäki, "Micro-operators to boost local service delivery in 5G," Wireless Personal Communications, vol. 95, pp. 69-82, 2017.

[10] M. Matinmikko, M. Latva-aho, P. Ahokangas \& V. Seppänen, "On regulations for 5G: Micro licensing for locally operated networks," Telecommunications Policy, to be published.

[11] M. Matinmikko, A. Roivainen, M. Latva-aho, and K. Hiltunen, "Interference study of micro licensing for 5G micro operator small cell deployments," Conference on Cognitive Radio Oriented Wireless Networks and Communications (CrownCom), Lisboa, Portugal, 20-21 September 2017.

[12] K. B. Shashika Manosha, M. Matinmikko, and M. Latva-aho, "Framework for spectrum authorization elements and its application to 5G micro-operators," Internet of Things Business Models, Users, and Networks, Copenhagen. Denmark, 23-24 November 2017, pp. 1-8.

[13] G. Biczok, M. Dramitinos, L. Toka, P. E. Heegaard, and H. Lonsethagen, "Manufactured by software: SDN-enabled multi-operator composite services with the 5G exchange," IEEE Communications Magazine, vol. 55, pp. 80-86, 2017.

[14] 3GPP. Study on management and orchestration of network slicing for next generation network. 3GPP TR 28.801 version 1.1.0. $3^{\text {rd }}$ Generation Partnership Project. 2017.

[15] ETSI. Network functions virtualisation (NFV); Management and orchestration; Functional requirements specification. GS NFV-IFA 010 V2.2.1. European Telecommunications Standards Institute. 2016.

[16] 3GPP. Study on new radio access technology: Radio access architecture and interfaces. 3GPP TR 38.801 version 14.0.0. $3^{\text {rd }}$ Generation Partnership Project. 2017.

[17] CEPT. Licensed shared access. ECC Report 205. European Conference of Postal and Telecommunications Administrations. 2014.

[18] FCC. Order on reconsideration and second report and order. Amendment of the Commission's rules with regard to commercial operations in the 3550-3650 MHz Band. FCC 16-55. Federal Communications Commission. 2016.

[19] A. Roivainen, C. Ferreira Dias, N. Tervo, V. Hovinen, M. Sonkki, and M. Latva-aho, "Geometry-based stochastic channel model for two-story lobby environment at $10 \mathrm{GHz}$," IEEE Transactions on Antennas and Propagation, vol. 64, no. 9, pp. 3990-4003, 2016. 